\title{
THE MAXIMAL VARIATION OF A BOUNDED MARTINGALE
}

\author{
BY \\ JEAN-FRANCOIS MERTENS AND SHMUEL ZAMIR
}

\section{ABSTRACT}

Let $\chi_{0}^{n}=\left\{X_{3}\right\}_{0}^{n}$ be a martingale such that $0 \leqq X_{1} \leqq 1 ; i=0, \cdots, n$. For $0 \leqq p \leqq 1$ denote by $\mathcal{M}_{p}^{n}$ the set of all such martingales satisfying also $E\left(X_{0}\right)=p$. The variation of a martingale $\chi_{0}^{n}$ is denoted by $V\left(\chi_{0}^{n}\right)$ and defined by $V\left(\chi_{0}^{n}\right)=$ $E\left(\sum_{r=0}^{n-1}\left|X_{1+1}-X_{i}\right|\right)$. It is proved that

$$
\lim _{n \rightarrow \infty}\left\{\operatorname{Sup}_{x_{0}^{n} \in \mathcal{M}_{p}^{n}}\left[\frac{1}{\sqrt{n}} V\left(\chi_{0}^{n}\right)\right]\right\}=\phi(p),
$$

where $\phi(p)$ is the well known normal density evaluated at its $p$-quantile, i.e.

$$
\phi(p)=\frac{1}{\sqrt{2 \pi}} \exp \left(-\frac{1}{2} x_{p}^{2}\right) \text { where } \int_{-\alpha}^{x_{p}} \frac{1}{\sqrt{2 \pi}} \exp \left(-\frac{1}{2} x^{2}\right) d x=p .
$$

A sequence of martingales $\chi_{0}^{n}, n=1,2, \cdots$ is constructed so as to satisfy $\lim _{n \rightarrow \infty}(1 / \sqrt{n}) V\left(\chi_{0}^{\eta}\right)=\phi(p)$.

\section{Introduction}

For a martingale $\chi_{0}^{n}=\left\{X_{i}\right\}_{0}^{n}$ we define the variation by $V\left(\chi_{0}^{n}\right)=$ $E\left(\sum_{t=0}^{n-1}\left|X_{t+1}-X_{t}\right|\right)$. We are interested in this variation for bounded martingales, say $0 \leqq X_{t} \leqq 1, i=0,1,2, \cdots$. For any $p: 0 \leqq p \leqq 1$ denote by $\mathcal{M}_{p}^{n}$ the set of all $n$-martingales bounded in $[0,1]$ and satisfying $E\left(X_{0}\right)=p(E(X)$ denotes the expectation of $X$ ).

A rather easy consequence of a well known property of martingales and the Cauchy-Schwartz inequality is that

$$
V\left(\chi_{0}^{n}\right) \leqq \sqrt{p(1-p)} \cdot \sqrt{n}
$$

for every $\chi_{0}^{n} \in \mathcal{M}_{p}^{n}$. In particular if $\left\{X_{t}\right\}_{0}^{\infty}$ is an infinite martingale with $E\left(X_{0}\right)=p$ and $\chi_{0}^{n}$ is its truncation at stage $n$, then (1.1) holds for $n=1,2, \cdots$. However this 
is not the strongest statement possible in this case since from the convergence of $\left\{X_{i}\right\}_{0}^{\infty}$ it can be shown that for any such $\infty$-martingale

$$
\lim _{n \rightarrow \infty} \frac{1}{\sqrt{n}} V\left(\chi_{0}^{n}\right)=0 .
$$

The question we are interested in is: Is $\sqrt{n}$ the least upper bound for the order of magnitude of $V\left(\chi_{n}^{n}\right)$ ? Since obviously there are $n$-martingales with $V\left(\chi_{0}^{n}\right)$ of lower order of magnitude, the question is: Is there a function $f(p) ; f(p)>0$ for $0<p<1$; such that for each $0 \leqq p \leqq 1$ and a positive integer $n$ there exists an $n$-martingale $\chi_{0}^{n} \in \mathcal{M}_{p}^{n}$ satisfying

$$
V\left(\chi_{0}^{n}\right) \geqq f(p) \sqrt{n} ?
$$

Notice that in view of (1.2) it is impossible to satisfy (1.3) with the $\chi_{0}^{n}$ being the truncations of the same $\infty$-martingale. An affirmative answer to the above stated question would imply: There exists $f(p): f(0)=f(1)=0$ and $f(p)>0$ for $0<p<1$ such that

$$
f(p) \leqq \operatorname{Sup}_{\chi_{n}^{n} \in \mathcal{M}_{p}^{n}}\left[\frac{1}{\sqrt{n}} V\left(\chi_{0}^{n}\right)\right] \leqq \sqrt{p(1-p)}
$$

It turns out that a result much stronger than (1.4) can be achieved, namely

$$
\lim _{n \rightarrow \infty}\left\{\operatorname{Sup}_{\chi_{0}^{n} \in \mathcal{M}_{p}^{n}}\left[\frac{1}{\sqrt{n}} V\left(\chi_{0}^{n}\right)\right]\right\}=\phi(p)
$$

where

$$
\phi(p)=\frac{1}{\sqrt{2 \pi}} \exp \left(-\frac{1}{2} x_{p}^{2}\right) ; \quad \int_{-\infty}^{x_{p}} \frac{1}{\sqrt{2 \pi}} \exp \left(-\frac{1}{2} x^{2}\right) d x=p
$$

Thus, not only is $\operatorname{Sup}_{x_{0}^{n} \in \mathcal{M}_{p}^{n}}\left[(1 / \sqrt{n}) V\left(\chi_{0}^{n}\right)\right]$ bounded away from 0 but it is a converging sequence, the limit of which is, amazingly enough, the well known normal density function evaluated at its $p$-quantile.

A by-product of the proof of this result is a construction of a sequence of martingales $\chi_{0}^{n} \in \mathcal{M}_{p}^{n} ; n=1,2, \cdots$ for which

$$
\lim _{n \rightarrow \infty}\left[\frac{1}{\sqrt{n}} V\left(\chi_{0}^{n}\right)\right]=\phi(p) \text {. }
$$

Our interest in the variation of bounded martingales came up through game theory. It turns out that the speed of convergence of the values of certain 
repeated games with incomplete information is given by $(1 / n) V\left(\chi_{0}^{n}\right)$ for some $\chi_{0}^{n} \in \mathcal{M}_{p}^{n}$. Using the results of this paper one can find a sequence of such games with asymptotic value 0 , for which the values $v_{n}(p)$ satisfy $\lim _{n \rightarrow \infty} \sqrt{n} v_{n}(p)=$ $\phi(p)$. These are in some sense repeated games with the slowest rate possible for releasing information (see [3]).

We are indebted to David Gilat for calling our attention to an inaccuracy in the first version of the paper.

\section{Preliminary results and statement of the main theorem}

We are considering $n$-martingales and $\infty$-martingales bounded in $[0,1]$ with a given and fixed expectation $p$, i.e., $\left\{X_{1}\right\}_{0}^{n}$ and $\left\{X_{1}\right\}_{0}^{\infty}$ such that $0 \leqq X_{1} \leqq 1 \forall i$ and $E\left(X_{0}\right)=p ; 0 \leqq p \leqq 1$ (hence also $\left.E\left(X_{i}\right)=p \forall i\right)$. We denote such $n$-martingales by $\chi_{0}^{n}\left(\right.$ or $\left.\chi_{0}^{\infty}\right)$ and the set of all such martingales by $\mathcal{M}_{p}^{n}$ (or $\mathcal{M}_{p}^{\infty}$ ).

DEFINITION 2.1 The $n$-stage variation of a martingale $\chi_{0}^{\infty}$ (or $\chi_{0}^{m}$ for $m \geqq n$ ) is denoted by $V\left(\chi_{0}^{n}\right)$ and defined by

$$
V\left(\chi_{0}^{n}\right)=E\left(\sum_{i=0}^{n-1}\left|X_{t+1}-X_{i}\right|\right)
$$

The following two Theorems may be partially or fully known. However, we state and prove them here for the sake of completeness and mainly to clarify the significance of our main result (Theorem 2.5).

THEOREM 2.2 For all $p, 0 \leqq p \leqq 1$ and $n=1,2, \cdots$

$$
\frac{1}{\sqrt{n}} V\left(\chi_{0}^{n}\right) \leqq \sqrt{p(1-p)}
$$

holds for all $\chi_{0}^{n} \in \mathcal{M}_{p}^{n}$.

Proof. We recall that since martingale differences are uncorrelated (see e.g. [1]), it follows that

$$
E\left(\sum_{0}^{n-1}\left(X_{t+1}-X_{t}\right)^{2}\right)=E\left(X_{n}-X_{0}\right)^{2}
$$

Since in our case the martingale is bounded in $[0,1]$ and $E\left(X_{0}\right)=p$, it is easily seen that the maximal value for $E\left(X_{n}-X_{0}\right)^{2}$ is attained when $X_{0} \equiv p$ and the distribution of $X_{n}$ is $\operatorname{Pr}\left(X_{n}=1\right)=p ; \operatorname{Pr}\left(X_{n}=0\right)=1-p$ in which case we have

$$
E\left(X_{n}-X_{0}\right)^{2}=E\left(X_{n}^{2}\right)-p^{2}=p-p^{2}=p(1-p) .
$$


Hence, by (2.2)

$$
E\left(\sum_{i=0}^{n-1}\left(X_{i+1}-x_{i}\right)^{2}\right) \leqq p(1-p) .
$$

Now we make use of the Cauchy-Schwartz inequality and get (using (2.3))

$$
\begin{aligned}
V\left(\chi_{0}^{n}\right)=E\left(\sum_{i=0}^{n-1}\left|X_{i+1}-X_{i}\right|\right) & \leqq\left[\left(\sum_{i=0}^{n-1}\left(X_{i+1}-X_{t}\right)^{2}\right) E\left(\sum_{i=0}^{n-1} 1^{2}\right)\right]^{1 / 2} \\
& \leqq \sqrt{p(1-p)} \cdot \sqrt{n},
\end{aligned}
$$

which completes the proof.

COROLlaRy 2.3 For any infinite martingale $\chi_{0}^{\infty}$ bounded in $[0,1]$ with $E\left(X_{0}\right)=p$ the $n$-stage variation is bounded by $\sqrt{p(1-p)} \cdot \sqrt{n}$ and consequently $0 \leqq \lim _{n \rightarrow \infty} \sup V\left(\chi_{0}^{n}\right) / \sqrt{n} \leqq \sqrt{p(1-p)}$.

However, as far as infinite martingales are concerned a stronger result can be obtained, namely

THEOREM 2.4. For any $\infty$-martingale $\chi_{0}^{\infty}$ with $0 \leqq X_{1} \leqq 1 ; i=1,2, \cdots$,

$$
\lim _{n \rightarrow \infty} \frac{1}{\sqrt{n}} V\left(\chi_{0}^{n}\right)=0
$$

Before proving this theorem let us notice that there is no hope to strengthen it so much as to prove that $V\left(\chi_{0}^{\infty}\right)<\infty$. In fact the following example communicated to us by David Gilat shows that a bounded martingale may have an infinite variation.

ExAmple. Perform a symmetric random walk $\left(p=\frac{1}{2}\right)$ with $X_{0} \equiv \frac{1}{2}$ and a step size $\frac{1}{8}$. Reduce the step size to $\frac{1}{32}$ as soon as you reach one of the points $\frac{1}{2} \pm \frac{1}{4}$. In general if the step size was last time reduced at point $y$ to size $\varepsilon_{k}=2^{-(2 k+1)}$, then reduce it to $\varepsilon_{k+1}=2^{-(2 k+3)}$ as soon as you reach one of the points $y \pm n_{k} \varepsilon_{k}$ where $n_{k}=2^{k}$. Doing that for $k=1,2, \cdots$ we obtain a martingale $\chi_{0}^{\infty}$ bounded in $[0,1]$ (since $\sum_{k=1}^{\infty} n_{k} \varepsilon_{k}=\frac{1}{2}$ ). If we denote by $N_{k}$ the number of steps of size $\varepsilon_{k}$ made, then clearly

$$
V\left(\chi_{0}^{\infty}\right)=\sum_{k=1}^{\infty} \varepsilon_{k} E\left(N_{k}\right)
$$

But $E\left(N_{k}\right)=n_{k}^{2}$ (expected duration of a classical ruin game, see e.g. [2] pp. 348), hence, 


$$
V\left(\chi_{0}^{\infty}\right)=\sum_{k=1}^{\infty} \varepsilon_{k} n_{k}^{2}=\sum_{k=1}^{\infty} 2^{-(2 k+1)} 2^{2 k}=\infty .
$$

ProOF OF THEOREM 2.4. Let $\chi_{0}^{\infty}=\left\{X_{n}\right\}_{0}^{\infty}$ be an $\infty$-martingale bounded in $[0,1]$. By (2.2), $\chi_{0}^{\infty}$ is (uniformly) bounded in $L_{2}$, hence $\chi_{0}^{\infty}$ converges in $L_{2}$ to a random variable say $X_{\infty}$. So $\forall \varepsilon>0, \exists k$ s.t. $\left\|X_{n}-X_{k+n}\right\|_{2}<\varepsilon$ for all $n$. Now

$$
\begin{aligned}
\frac{1}{\sqrt{k+n}} V\left(\chi_{0}^{k+n}\right) & =\frac{1}{\sqrt{k+n}} \sum_{i=0}^{k+n-1} E\left(\left|X_{i+1}-X_{i}\right|\right) \\
& =\frac{1}{\sqrt{k+n}}\left[\sum_{i=0}^{k-1} E\left(\left|X_{i+1}-X_{i}\right|\right)+\sum_{i=k}^{k+n-1} E\left(\left|X_{i+1}-X_{i}\right|\right)\right] .
\end{aligned}
$$

Using the Cauchy-Schwartz inequality for each of the two sums and then applying (2.2) we get

$$
\begin{aligned}
\frac{1}{\sqrt{k+n}} V\left(\chi_{0}^{k+n}\right) \leqq & \frac{\sqrt{k}}{\sqrt{k+n}}\left[\sum_{i=0}^{k-1} E\left(X_{i+1}-X_{i}\right)^{2}\right]^{1 / 2} \\
& +\frac{\sqrt{n}}{\sqrt{k+n}}\left[\sum_{i=k}^{k+n-1} E\left(X_{i+1}-X_{i}\right)^{2}\right]^{1 / 2} \\
\leqq & \frac{\sqrt{k}}{\sqrt{k+n}}\left\|X_{k}-X_{0}\right\|_{2}+\frac{\sqrt{n}}{\sqrt{k+n}}\left\|X_{k+n}-X_{k}\right\|_{2} .
\end{aligned}
$$

Letting $n \rightarrow \infty$ we conclude that $\forall \varepsilon>0 \exists k$ s.t

$$
\lim _{n \rightarrow \infty} \frac{1}{\sqrt{k+n}} V\left(\chi_{0}^{k+n}\right)<\varepsilon,
$$

in other words

$$
\lim _{n \rightarrow \infty} \frac{1}{\sqrt{n}} V\left(\chi_{0}^{n}\right)=0
$$

as claimed. We now turn to the main result of the paper:

THEOREM 2.5 (The Main Theorem). For any $p ; 0 \leqq p \leqq 1$ :

$$
\lim _{n \rightarrow \infty}\left\{\operatorname{Sup}_{\chi \tilde{x} \in \mathcal{M}_{p}^{n}}\left[\frac{1}{\sqrt{n}} V\left(\chi_{0}^{n}\right)\right]\right\}=\phi(p)
$$

where $\phi(p)$ is the standard normal density function evaluated at its $p$-quantile (i.e. $\phi(p)=(1 / \sqrt{2 \pi}) \exp \left(-\frac{1}{2} x_{p}^{2}\right)$ where $\left.\int_{-\infty}^{x_{p}}(1 / \sqrt{2 \pi}) \exp \left(-\frac{1}{2} x^{2}\right) d x=p\right)$. 
In view of the length and the technical complication of the proof we provide in the next section the heuristic arguments that lead, and in fact have led us, to the result. The formal proof is given in section 4 .

\section{The heuristics of the result}

Let

$$
\phi_{n}(p)=\operatorname{Sup}_{\chi \chi_{0}^{n} \in \mathcal{M}_{p}^{n}}\left[\frac{1}{\sqrt{n}} V\left(\chi_{0}^{n}\right)\right] .
$$

Assuming $X_{0} \equiv p, \phi_{n}(p)$ clearly satisfies the following recursion formula:

$$
\sqrt{n+1} \phi_{n+1}(p)=\operatorname{Max}_{\left\{X_{1} \mid E\left(X_{1}\right)=p\right\}}\left\{E\left(\left|X_{3}-p\right|\right)+\sqrt{n} E\left(\phi_{n}\left(X_{1}\right)\right)\right\}
$$

$$
\begin{aligned}
=\operatorname{Max}_{\left\{X_{1} \mid E\left(X_{1}\right)=p\right\}}\left\{P\left(X_{1} \geqq p\right)\right. \\
\quad \times\left[E\left(X_{1}-p \mid X_{1} \geqq p\right)+\sqrt{n} E\left(\phi_{n}\left(X_{1}\right) \mid X_{1} \geqq p\right)\right] \\
\left.+P\left(X_{1}<p\right)\left[E\left(p-X_{1} \mid X_{1}<p\right)+\sqrt{n} E\left(\phi_{n}\left(X_{1}\right) \mid X_{1}<p\right)\right]\right\} .
\end{aligned}
$$

Assuming that $\phi_{n}$ is concave (an assumption based on observation of $\phi_{1}$ and $\phi_{2}$ ), then for any $X_{1}$, the expression to be maximized in (3.1) is increased if $X_{1}$ is replaced by $\tilde{X}_{1}$ which assumes only the two values $p+\xi$ and $p-\eta$, where $\xi=E\left(X_{1}-p \mid X_{1} \geqq p\right)$ and $\eta=E\left(p-X_{1} \mid X_{1}<p\right)$. Since in addition $E\left(X_{1}\right)=p$ we replace (3.1) by

$$
\sqrt{n+1} \phi_{n+1}(p)=\operatorname{Max}_{(\xi, \eta) \in S(p)}\left\{\sqrt{n}\left[\frac{\eta}{\xi+\eta} \phi_{n}(p+\xi)+\frac{\xi}{\xi+\eta} \phi_{n}(p-\eta)\right]+\frac{2 \xi \eta}{\xi+\eta}\right\}
$$

where $S(p)=\{(\xi, \eta) \mid 0 \leqq \xi \leqq 1-p ; 0 \leqq \eta \leqq p\}$.

By concavity of $\phi_{n}$ the expression in the square brackets decreases both in $\xi$ and in $\eta$. Since this expression is multiplied by $\sqrt{n}$, it follows that the points $\left(\xi_{n}, \eta_{n}\right)$ at which (3.2) achieves its maximum, satisfy $\left(\xi_{n}, \eta_{n}\right) \underset{n \rightarrow \infty}{\longrightarrow}(0,0)$. Expanding $\phi_{n}(p+\xi)$ and $\phi_{n}(p-\eta)$ we obtain the following approximation:

$$
\sqrt{n+1} \phi_{n+1}(p) \cong\left\{\sqrt{n}\left[\phi_{n}(p)+\xi_{n} \eta_{n} \phi_{n}^{\prime \prime}(p)\right]+2 \xi_{n} \eta_{n} /\left(\xi_{n}+\eta_{n}\right)\right\}
$$

For any fixed $\xi_{n} \eta_{n}$ the expression at the right hand side of (3.3) is maximized when $\left(\xi_{n}+\eta_{n}\right)$ is minimized, which is at $\xi_{n}=\eta_{n}$. We conclude that as a first approximation, the maximum in (3.2) is achieved for $\xi=\eta$. Motivated by this 
observation we restrict the domain of maximization $S(p)$ in (3.2) to $S(p) \cap\{\xi=$ $\eta\}=\left\{x \mid 0 \leqq x \leqq p^{*}\right\}$, where $p^{*}=\min (p, 1-p)$. The recursion equation (3.2) is thus replaced by

$$
\sqrt{n+1} \phi_{n+1}(p)=\operatorname{Max}_{0 \leq x \leq p^{*}}\left\{\frac{1}{2} \sqrt{n}\left[\phi_{n}(p+x)+\phi_{n}(p-x)\right]+x\right\}
$$

Assuming now that $\phi_{n}(p)$ converge to some function $\varphi(p)$ one gets from (3.4), letting $x=\alpha_{n} / \sqrt{n}$,

$$
\begin{aligned}
\sqrt{1+\frac{1}{n}} \varphi(p) & \cong \operatorname{Max}_{\alpha_{n}}\left\{\frac{\alpha_{n}}{n}+\frac{1}{2}\left[\varphi\left(p+\alpha_{n} / \sqrt{n}\right)+\varphi\left(p-\alpha_{n} / \sqrt{n}\right)\right]\right\} \\
& \cong \operatorname{Max}_{\alpha_{n}}\left\{\frac{\alpha_{n}}{n}+\varphi(p)+\frac{\alpha_{n}^{2}}{2 n} \varphi^{\prime \prime}(p)\right\} \\
& \cong \varphi+\frac{1}{n} \operatorname{Max}_{\alpha_{n}}\left\{\alpha_{n}+\frac{\alpha_{n}^{2}}{2} \varphi^{\prime \prime}\right\}=\varphi-\frac{1}{2 n \varphi^{\prime \prime}} .
\end{aligned}
$$

On the other hand $\sqrt{1+1 / n} \varphi \cong \varphi+(1 / 2 n) \varphi$, thus $\varphi=-1 / \varphi^{\prime \prime}$. In other words we are led to the differential equation

$$
\varphi \varphi^{\prime \prime}+1=0 .
$$

To solve (3.5) we rewrite it as $-\varphi^{\prime}(p)=\int_{1 / 2}^{p}(1 / \varphi) d p$, where we have introduced an integration constant so as to have $\varphi^{\prime}\left(\frac{1}{2}\right)=0$ which is implied by the symmetry of $\varphi(p)$ around $p=\frac{1}{2}$. Now let $z(p)=-\varphi^{\prime}(p)=\int_{1 / 2}^{p}(1 / \varphi) d p$, then $z^{\prime}(p)=1 / \varphi$ and thus $\varphi=d p / d z$. Now replace in (3.5) the variable $p$ by the variable $z$ :

$$
\varphi_{z}^{\prime}=\varphi_{p}^{\prime} \frac{d p}{d z}=\varphi_{p}^{\prime} \varphi=-z \varphi,
$$

which implies $\ln \varphi=K-\frac{1}{2} z^{2}$ or

$$
\varphi=A \frac{1}{\sqrt{2 \pi}} \exp \left(-\frac{1}{2} z^{2}\right)
$$

(where $K$ and $A$ are constants). Since $\varphi=d p / d z$ we get

$$
p=c+\int_{-\infty}^{z(p)} A \frac{1}{\sqrt{2 \pi}} \exp \left(-\frac{1}{2} x^{2}\right) d x .
$$

Denoting by $F(x)$ the cumulative standard normal distribution we have therefore 


$$
\begin{aligned}
\varphi(z) & =A F^{\prime}(z(p)), \\
p & =c+A F(z(p)) .
\end{aligned}
$$

Now $\varphi \geqq 0$ and $\varphi \not \equiv 0$ implies $A>0$ from which it follows by (3.7) that $z(p)$ is monotonously increasing with $p$. Since $\varphi(0)=\varphi(1)=0$ we have by (3.6) that $z(0)=-\infty, z(1)=+\infty$. From (3.7) we thus have

$$
1=c+A \text {. }
$$

From $\varphi^{\prime}\left(\frac{1}{2}\right)=0$ we get $z\left(\frac{1}{2}\right)=-\varphi^{\prime}\left(\frac{1}{2}\right)=0$, hence from (3.7)

$$
\frac{1}{2}=c+\frac{1}{2} A \text {. }
$$

We conclude from (3.10) and (3.11) that $c=0$ and $A=1$, thus finally $\varphi(p)=$ $F^{\prime}(z), p=F(z)$, i.e. the limit $\varphi(p)$ is the standard normal density evaluated at its $p$-quantile.

\section{Proof of the Main Theorem}

First let us introduce the convention of writing $p^{\prime}$ instead of $1-p$ for $0 \leqq p \leqq 1$. (Although we will use the prime also for derivative it will be clear from the context which operation it stands for.) Next, for $0 \leqq p \leqq 1$ let

$$
S(p)=\left\{(\xi, \eta){\substack{0 \leq \xi \leq p^{\prime} \\ 0 \leq \eta \leq p}}_{\substack{0 \\ 0}}\right.
$$

and define two sequences of functions on $[0,1],\left\{\varphi_{n}\right\}$ and $\left\{\psi_{n}\right\}$ by $\varphi_{0} \equiv \psi_{0} \equiv 0$ and for $n=0,1,2, \cdots$

$$
\begin{aligned}
& \sqrt{n+1} \varphi_{n+1}(p)=\operatorname{Max}_{(\xi, n) \in S(p)}[\left.\sqrt{n} \frac{\eta}{\xi+\eta} \psi_{n}(p+\xi)+\sqrt{n} \frac{\xi}{\xi+\eta} \psi_{n}(p-\eta)+\frac{2 \xi \eta}{\xi+\eta}\right], \\
& \psi_{n+1}=\operatorname{Cav} \varphi_{n+1} .
\end{aligned}
$$

In (4.1) the expression in the square brackets is defined to be $\psi_{n}(p)$ when $\xi=\eta=0$. In (4.2) Cav is the operator of concavification of a function on $[0,1]$, (i.e. Cav $f$ is the smallest concave function $g$ satisfying $g(p) \geqq f(p) ; 0 \leqq p \leqq 1$ ).

We first observe that

$$
\varphi_{n}(0)=\psi_{n}(0)=\varphi_{n}(1)=\psi_{n}(1)=0 \text { for } n=0,1,2, \cdots .
$$

It also follows immediately from the definitions that

$$
\varphi_{1}(p)=\psi_{1}(p)=2 p(1-p) \text { for } 0 \leqq p \leqq 1 .
$$

LEMMA 4.1. Every $\varphi_{n}$ and every $\psi_{n}, n=0,1,2, \cdots$ is symmetric around $p=\frac{1}{2}$. 
This is easily proved by induction using (4.1) and observing that the operation Cav conserves the symmetry around $p=\frac{1}{2}$.

LEMma 4.2.

$$
\operatorname{Sup}_{\chi \chi_{0}^{1} \in \mathcal{M}_{p}^{\prime}} V\left(\chi_{0}^{1}\right)=\psi_{1}(p)=2 p(1-p)
$$

Proof. For any martingale $\chi_{0}^{n} \in \mathcal{M}_{p}^{n}$ we denote by $V\left(\chi_{0}^{n} \mid X_{0}=\alpha\right)$ the conditional $n$-stage variation given the value $\alpha$ of $X_{0}$. We shall first show

$$
\operatorname{Sup}_{X_{1}} V\left(\chi_{0}^{1} \mid X_{0}=\alpha\right)=2 \alpha(1-\alpha),
$$

where the Sup is being taken over all r.v. (random variables) in [0,1] s.t. $E\left(X_{1}\right)=\alpha$. To prove (4.6) take any such $X_{1}$ and let $\Omega_{1}=\left\{X_{1}>\alpha\right\}, \Omega_{2}=\left\{X_{1} \leqq \alpha\right\}$, then

$$
\begin{aligned}
V\left(\chi_{0}^{1} \mid X_{0}=\alpha\right) & =E\left(\left|X_{1}-\alpha\right|\right) \\
& =P\left(\Omega_{1}\right) E\left(\left(X_{1}-\alpha\right) \mid \Omega_{1}\right)+P\left(\Omega_{2}\right) E\left(\left(\alpha-X_{1}\right) \mid \Omega_{2}\right) \\
& =P\left(\Omega_{1}\right) \cdot \xi+\left(1-P\left(\Omega_{1}\right)\right) \cdot \eta
\end{aligned}
$$

where $\xi=E\left(\left(X_{1}-\alpha\right) \mid \Omega_{1}\right)$ and $\eta=E\left(\left(\alpha-X_{1}\right) \mid \Omega_{2}\right)$. Now

$$
\begin{aligned}
\alpha & =E\left(X_{1}\right)=P\left(\Omega_{1}\right) E\left(X_{1} \mid \Omega_{1}\right)+\left(1-P\left(\Omega_{1}\right)\right) E\left(X_{1} \mid \Omega_{2}\right) \\
& =P\left(\Omega_{1}\right)(\alpha+\xi)+\left(1-P\left(\Omega_{1}\right)\right)(\alpha-\eta),
\end{aligned}
$$

which implies $P\left(\Omega_{1}\right)=\eta /(\xi+\eta)$ and hence $V\left(\chi_{0}^{1} \mid X_{0}=\alpha\right)=2 \xi \eta /(\xi+\eta)$. Therefore

$$
\operatorname{Sup} V\left(\chi_{0}^{1} \mid X_{0}=\alpha\right)=\operatorname{Sup}_{(\xi, \eta) \in S(\alpha)} 2 \xi \eta /(\xi+\eta) .
$$

In (4.7) the sup is achieved for $\xi=\alpha^{\prime}=1-\alpha$ and $\eta=\alpha$, which establishes (4.6).

Now if we denote by $E_{X_{0}}$ the expectation with respect to the r.v. $X_{0}$ we have

$$
\begin{aligned}
\operatorname{Sup}_{\chi_{0}^{1} \in \mathcal{M}_{p}^{1}} V\left(\chi_{0}^{1}\right) & =\operatorname{Sup}_{\left\{X_{0} \mid E\left(X_{0}\right)=p\right\}} E_{X_{0}}\left[\operatorname{Sup}_{X_{1}} V\left(\chi_{0}^{1} \mid X_{0}\right)\right] \\
& =\operatorname{Sup}_{\left\{X_{0} \mid E\left(X_{0}\right)=p\right\}} E\left(2 X_{0}\left(1-X_{0}\right)\right) .
\end{aligned}
$$

Finally since $2 X_{0}\left(1-X_{0}\right)$ is a concave function w.r.t. $X_{0}$, the $\operatorname{Sup}_{\left\{X_{0} \mid E\left(X_{0}\right)=p\right\}}$ is achieved for $X_{0} \equiv p$ which concludes the proof of Lemma 4.2.

Theorem 4.3. For $n=0,1,2, \cdots$ and $0 \leqq p \leqq 1$ 


$$
\operatorname{Sup}_{\chi_{0}^{n} \in \mathscr{M}_{p}^{n}}\left[\frac{1}{\sqrt{n}} V\left(\chi_{0}^{n}\right)\right] \leqq \psi_{n}(p)
$$

Proof. By induction on $n$ : For $n=1,(4.8)$ follows from Lemma 4.2. Assume (4.8) is true for $n \leqq m-1$ and let us prove it for $n=m$. Since $\psi_{m}=\operatorname{Cav} \varphi_{m}$ it is clearly enough to prove that for $0 \leqq p \leqq 1$

$$
\operatorname{Sup}_{\substack{x^{m} \in \mathcal{M}_{p}^{m} \\ X_{0}=p}} V\left[\frac{1}{\sqrt{m}} V\left(\chi_{0}^{m}\right)\right] \leqq \varphi_{m}(p) .
$$

To prove (4.9) let $\Omega_{1}=\left\{x_{1}>p\right\}, \Pi=P\left(\Omega_{1}\right)$ (hence $1-\Pi=P\left(\Omega_{2}\right)$ ). We have for any $\left\{X_{1}\right\}_{0}^{m}, X_{0} \equiv p$

$$
\begin{aligned}
V\left(\chi_{0}^{m}\right) & =\sum_{i=1}^{m-1} E\left(\left|X_{1}-X_{t-1}\right|\right) \\
& =E\left(\left|X_{0}-p\right|\right)+\Pi \sum_{i=2}^{m-1} E\left(\mid X_{1}-X_{1-1} \| \Omega_{1}\right)+(1-\Pi) \sum_{i=2}^{m-1} E\left(\mid X_{i}-X_{t-1} \| \Omega_{2}\right)
\end{aligned}
$$

(by induction hypothesis)

$$
\begin{aligned}
\leqq & E\left(\left|X_{1}-p\right|\right)+\sqrt{m-1}\left[\Pi \psi_{m-1}\left(E\left(X_{1} \mid \Omega_{1}\right)\right)+(1-\Pi) \psi_{m-1}\left(E\left(X_{1} \mid \Omega_{2}\right)\right)\right] \\
= & \Pi\left(E\left(X_{1} \mid \Omega_{1}\right)-p\right)+(1-\Pi)\left(p-E\left(X_{1} \mid \Omega_{2}\right)\right) \\
& +\sqrt{m-1}\left[\Pi \psi_{m-1}\left(E\left(X_{1} \mid \Omega_{1}\right)\right)+(1-\Pi) \psi_{m-1}\left(E\left(X_{1} \mid \Omega_{2}\right)\right)\right] .
\end{aligned}
$$

Let $p+\xi=E\left(X_{1} \mid \Omega_{1}\right)$ and $p-\eta=E\left(X_{1} \mid \Omega_{2}\right)$, then $\xi \geqq 0, \quad \eta \geqq 0$ and $\Pi \xi-(1-\Pi) \eta=0$ which implies $\Pi=\eta /(\xi+\eta)$. From our last inequality we thus obtain

$$
V\left(\chi_{0}^{m}\right) \leqq \sqrt{m-1}\left[\frac{\eta}{\xi+\eta} \psi_{m-1}(p+\xi)+\frac{\xi}{\xi+\eta} \psi_{m-1}(p-\eta)\right]+\frac{2 \xi \eta}{\xi+\eta},
$$

and by definition of $\varphi_{m}(p): V\left(\chi_{0}^{m}\right) \leqq \sqrt{m} \varphi_{m}(p)$, which concludes the proof of Theorem 4.3.

The following two Lemmas provide bounds for the convexity of the function $\phi(p)$.

LEMMA 4.4. There exists a constant $c>0$ such that

$$
\frac{1}{\sqrt{n+1}} \operatorname{Max}_{0 \leq x \leq \min \left(p, p^{\prime}\right)}\left[\frac{\sqrt{n}}{2}(\phi(p+x)+\phi(p-x))+x\right] \geqq \phi(p)-c / n^{2}
$$

for all $0 \leqq p \leqq 1 ; n=1,2, \cdots$. 
LeMmA 4.5. There exists a constant $K>0$ s.t. for $0 \leqq p \leqq 1$

$$
\begin{aligned}
\frac{1}{\sqrt{n+1}} \operatorname{Max}_{(\xi, \eta) \in S(p)}\left[\sqrt{n} \frac{\eta}{\xi+\eta} \phi(p+\xi)+\sqrt{n} \frac{\xi}{\xi+\eta} \phi(p-\eta)\right. & \left.+\frac{2 \xi \eta}{\xi+\eta}\right] \\
& \leqq \phi(p)+K / n^{2}
\end{aligned}
$$

Unfortunately the proofs of these technical Lemmas about $\phi(p)$ are rather lengthy. They can be found in the Appendix. Proceeding in the proof of our main theorem we define now a new sequence $\left\{\tilde{\phi}_{n}\right\}_{0}^{\infty}$ of functions on $[0,1]$ by $\tilde{\phi}_{0} \equiv 0$ and

$$
\sqrt{n+1} \tilde{\phi}_{n+1}(p)=\operatorname{Max}_{(\xi, \eta) \in S(p)}\left[\sqrt{n} \frac{\eta}{\xi+\eta} \tilde{\phi}_{n}(p+\xi)+\sqrt{n} \frac{\xi}{\xi+\eta} \tilde{\phi}_{n}(p-\eta)+\frac{2 \xi \eta}{\xi+\eta}\right.
$$

(Here again the expression in the brackets is defined to be $\bar{\phi}_{n}(p)$ if $\xi=\eta=0$.)

Proposition 4.6.

$$
\psi_{n}(p) \geqq \bar{\phi}_{n}(p) \text { for } \quad 0 \leqq p \leqq 1 ; n=0,1,2, \cdots .
$$

This follows readily from the definitions (4.1) (4.2) and (4.12).

Lemma 4.7. For $n=1,2, \cdots$ and $0 \leqq p \leqq 1$

$$
\operatorname{Sup}_{\chi \overline{0} \in \mathcal{M}_{p}^{n}}\left[\frac{1}{\sqrt{n}} V\left(\chi_{0}^{n}\right)\right] \geqq \tilde{\phi}_{n}(p) .
$$

Proof. For each $n=1,2, \cdots$ let us construct for each $p, 0 \leqq p \leqq 1$ a martingale $\chi_{0}^{n}(p) \in \mathcal{M}_{p}^{n}$ with variation exactly $\tilde{\phi}_{n}(p)$, i.e.

$$
\frac{1}{\sqrt{n}} V\left(\chi_{0}^{n}(p)\right)=\tilde{\phi}_{n}(p) \text {. }
$$

We do the construction inductively on $n$ : For $n=1$ let $X_{0}(p) \equiv p$ and $\operatorname{Pr}\left\{X_{1}(p)=0\right\}=p^{\prime} ; \operatorname{Pr}\left\{X_{1}(p)=1\right\}=p$ then $V\left(\chi_{0}^{1}(p)\right)=2 p p^{\prime}=\tilde{\phi}_{1}(p)$.

Assume now that for $n$ and for each $p, 0 \leqq p \leqq 1$ there is a martingale $\left\{X_{i}(p)\right\}_{0}^{n}$ satisfying (4.15). Let $\left(\xi_{n}, \eta_{n}\right)$ be the point at which the maximum in (4.12) is attained. Define the martingale $\left\{Z_{i}(p)\right\}_{0}^{n+1}$ by

$$
\left\{\begin{array}{l}
Z_{0}(p) \equiv p ; \quad \operatorname{Pr}\left\{Z_{1}(p)=p+\xi_{n}\right\}=\eta_{n} /\left(\xi_{n}+\eta_{n}\right) \\
\operatorname{Pr}\left\{Z_{1}(p)=p-\eta_{n}\right\}=\xi_{n} /\left(\xi_{n}+\eta_{n}\right) \\
\left\{Z_{1}(p) \mid Z_{1}(p)=p+\xi_{n}\right\}_{2}^{n}=\left\{X_{i}\left(p+\xi_{n}\right)\right\}_{1}^{n} \\
\left\{Z_{1}(p) \mid Z_{1}(p)=p-\eta_{n}\right\}_{2}^{n}=\left\{X_{i}\left(p-\eta_{n}\right)\right\}_{1}^{n}
\end{array}\right.
$$


It follows by (4.12) and (4.16) that

$$
\begin{aligned}
& \frac{1}{\sqrt{n+1}} V\left(Z_{0}^{n+1}(p)\right)=\frac{1}{\sqrt{n+1}}\left[\frac{2 \xi_{n} \eta_{n}}{\xi_{n}+\eta_{n}}+\frac{\eta_{n}}{\xi_{n}+\eta_{n}} V\left(\chi_{0}^{n}\left(p+\xi_{n}\right)\right)\right. \\
& \left.+\frac{\xi_{n}}{\xi_{n}+\eta_{n}} V\left(\chi_{0}^{n}\left(p-\eta_{n}\right)\right)\right] \\
& =\frac{1}{\sqrt{n+1}}\left[\frac{2 \xi_{n} \eta_{n}}{\xi_{n}+\eta_{n}}+\frac{\eta_{n}}{\xi_{n}+\eta_{n}} \sqrt{n} \phi_{n}\left(p+\xi_{n}\right)+\frac{\xi_{n}}{\xi_{n}+\eta_{n}} \sqrt{n} \phi_{n}\left(p-\eta_{n}\right)\right] \\
& =\tilde{\phi}_{n+1}(p) \text {. }
\end{aligned}
$$

This completes the proof of Lemma 4.7.

LEMMA 4.8. For $0 \leqq p \leqq 1 ; n=1,2, \cdots$

$$
\tilde{\phi}_{n}(p) \geqq \phi(p)-\frac{\alpha}{\sqrt{n}},
$$

for some constant $\alpha>0$.

Proof. We first prove by induction on $k$ that for any $n \geqq 1$

$$
\tilde{\phi}_{n+k}(p) \geqq \phi(p)-\frac{1}{\sqrt{n+k}}\left[\frac{\sqrt{n}}{2}+\sum_{i=n}^{n+k} \frac{4 c}{i \sqrt{i}}\right]
$$

for $k=0,1,2, \cdots$ where $c$ is a constant satisfying (4.10). In fact for $k=0$, $\tilde{\phi}_{n}(p) \geqq 0 \geqq \phi(p)-\frac{1}{2}$. We notice that in (4.10) we may replace the last term $-c / n^{2}$ by $-4 c /(n+1)^{2}$. Assume now that $(4.18)$ holds for $k$, then by (4.10)

$$
\begin{aligned}
\tilde{\phi}_{n+k+1}(p)= & \frac{1}{\sqrt{n+k+1}} \operatorname{Max}_{(\xi, \eta) \in S(p)}\left[\sqrt{n+k} \frac{\xi}{\xi+\eta} \tilde{\phi}_{n+k}(p+\xi)+\right. \\
& \left.+\sqrt{n+k} \frac{\xi}{\xi+\eta} \tilde{\phi}_{n+k}(p-\eta)+\frac{2 \xi \eta}{\xi+\eta}\right] \\
\geqq & \frac{1}{\sqrt{n+k+1}} \operatorname{Max}_{0 \leq x \leq p \wedge p^{\prime}}\left\{\frac{\sqrt{n+k}}{2}\left(\tilde{\phi}_{n+k}(p+x)+\tilde{\phi}_{n+k}(p-x)\right)+x\right\} \\
\geqq & \frac{1}{\sqrt{n+k+1}} \operatorname{Max}_{0 \leq x \leq p \wedge p^{\prime}}\left\{\frac{\sqrt{n+k}}{2}[\phi(p+x)+\phi(p-x)+x\right. \\
& \left.\left.-\frac{2}{\sqrt{n+k}}\left(\frac{\sqrt{n}}{2}+\sum_{i=n}^{n+k} \frac{4 c}{i \sqrt{i}}\right)\right]\right\}
\end{aligned}
$$




$$
\begin{aligned}
& \geqq \phi(p)-\frac{4 c}{(n+k+1)^{2}}-\frac{1}{\sqrt{n+k+1}}\left(\frac{\sqrt{n}}{2}+\sum_{i=n}^{n+k} \frac{4 c}{i \sqrt{i}}\right) \\
& =\phi(p)-\frac{1}{\sqrt{n+k+1}}\left(\frac{\sqrt{n}}{2}+\sum_{i=n}^{n+k+1} \frac{4 c}{i \sqrt{i}}\right) .
\end{aligned}
$$

Now by (4.18) for $n=1$

$$
\tilde{\phi}_{k+1}(p) \geqq \phi(p)-\frac{1}{\sqrt{k+1}}\left(\frac{1}{2}+\sum_{i=1}^{\infty} \frac{4 c}{i \sqrt{i}}\right)=\phi(p)-\frac{\alpha}{\sqrt{k+1}} .
$$

Since this holds for $k=0,1, \cdots$, the proof of the Lemma is completed.

LEMMA 4.9. For $0 \leqq p \leqq 1$ and $n=1,2, \cdots$

$$
\psi_{n}(p) \leqq \phi(p)+\beta / \sqrt{n}
$$

for some constant $\beta>0$.

Proof. The proof is almost the same as that of Lemma 4.8. First $\psi_{1}(p)=$ $2 p p^{\prime} \leqq \phi(p)+\frac{1}{2}$. Next we use Lemma 4.5 and (4.1) to prove that

$$
\psi_{k+1} \leqq \phi(p)+\frac{1}{\sqrt{k+1}}\left(\frac{1}{2}+\sum_{i=1}^{k+1} \frac{K}{i \sqrt{i}}\right)
$$

implies

$$
\varphi_{k+2}(p) \leqq \phi(p)+\frac{1}{\sqrt{k+2}}\left(\frac{1}{2}+\sum_{i=1}^{k+2} \frac{K}{i \sqrt{i}}\right)
$$

$K$ is a constant satisfying (4.11).

Since the function on the right hand side of (4.20) is concave, (4.20) implies

$$
\psi_{k+2}(p)=\operatorname{Cav} \varphi_{k+2}(p) \leqq \phi(p)+\frac{1}{\sqrt{k+2}}\left(\frac{1}{2}+\sum_{i=1}^{k+2} \frac{K}{i \sqrt{i}}\right) .
$$

Hence we have proved (4.19) by induction for $k=0,1 \cdots$. Finally it follows from (4.19) that

$$
\psi_{k+1} \leqq \phi(p)+\frac{\beta}{\sqrt{k+1}} ; \quad k=0,1, \cdots,
$$

holds for some constant $\beta$. This completes the proof of the Lemma.

We are now in the position to conclude the proof of our main Theorem: 
Proof of Theorem 2.5. By Lemmas 4.7 and 4.8

$$
\lim _{n \rightarrow \infty}\left\{\operatorname{Sup}_{\chi \chi_{0}^{n} \in \mu_{p}^{n}}\left[\frac{1}{\sqrt{n}} V\left(\chi_{0}^{n}\right)\right]\right\} \geqq \lim \operatorname{Inf}_{n \rightarrow \infty} \tilde{\phi}_{n}(p) \geqq \phi(p)
$$

and by Theorem 4.3 and Lemma 4.9

$$
\operatorname{limSup}_{n \rightarrow \infty}\left\{\operatorname{Sup}_{\chi_{\hat{0}}^{n} \in \mathcal{M}_{p}^{n}}\left[\frac{1}{\sqrt{n}} V\left(\chi_{0}^{n}\right)\right]\right\} \leqq \operatorname{limSup}_{n \rightarrow \infty} \psi_{n}(p) \leqq \phi(p) .
$$

COROLLARY 4.10. The n-martingales $\chi_{0}^{n}(p)$ constructed in the proof of Lemma 4.7 satisfy

$$
\lim _{n \rightarrow \infty}\left[\frac{1}{\sqrt{n}} V\left(\chi_{0}^{n}(p)\right)\right]=\phi(p) ; \quad 0 \leqq p \leqq 1,
$$

with the speed of convergence of the order of $1 / \sqrt{n}$.

In fact by construction

$$
\frac{1}{\sqrt{n}} V\left(\chi_{0}^{n}(p)\right)=\tilde{\phi}_{n}(p) ; \quad 0 \leqq p \leqq 1 ; \quad n=1,2, \cdots
$$

while by Lemmas $4.8,4.9$, and Proposition 4.6

$$
\phi(p)-\alpha / \sqrt{n} \leqq \tilde{\phi}_{n}(p) \leqq \psi_{n}(p) \leqq \phi(p)+\beta / \sqrt{n}
$$

APPENDIX

\section{Proofs of Lemmas 4.4 and 4.5}

We intend to prove in this Appendix two technical statements (namely, Lemmas 4.4 and 4.5 ) about the function $\phi(p)$ defined on $0 \leqq p \leqq 1$ by

$$
\text { (A.1) } \phi(p)=\frac{1}{\sqrt{2 \pi}} \exp \left(-\frac{1}{2} x_{p}^{2}\right) \text { where } \int_{-\infty}^{x_{p}} \frac{1}{\sqrt{2 \pi}} \exp \left(-\frac{1}{2} x^{2}\right) d x=p \text {. }
$$

We start by examining the derivatives of $\phi(p)$ :

Proposition A.1. For $0 \leqq p \leqq 1$ and $\phi(p)$ and $x_{p}$ defined by (A.1)
(a) $\phi^{\prime}(p)=-x_{p}$,
(b) $x_{p}^{\prime}=1 / \phi(p)$,
(c) $\phi^{\prime \prime}(p)=-1 / \phi(p)=-x_{p}^{\prime}$,
(d) $\phi^{(3)}(p)=-x_{p} / \phi^{2}(p)$,
(e) $\phi^{(4)}(p)=-\left(1+2 x_{p}^{2}\right) / \phi^{3}(p)$, 
(f) $\phi^{(5)}(p)=-x_{p}\left(7+6 x_{p}^{2}\right) / \phi^{4}(p)$,

(g) $\phi^{(6)}(p)=-\left(4 x_{p}^{2}+7\right)\left(6 x_{p}^{2}+1\right) / \phi^{5}(p)$,

(h) $\quad \phi^{(2 n)}(p) \leqq 0 ; n=1,2, \cdots$.

Proof. (a) to (g) result from straightforward differentiation. (h) will follow if we prove that

$$
\phi^{(2 n)}(p)=\frac{-1}{\phi^{2 n-1}(p)} \sum_{j=0}^{n-1} a_{1} x_{p}^{2 \prime}
$$

where $a_{1} \geqq 0$ for $j=1, \cdots, n-1$.

We prove (A.2) by induction on $n$. By (c) it is true for $n=1$. Assume it for $n$, then

$$
\begin{aligned}
\phi^{(2 n+1)}(p) & =\frac{-1}{\phi^{2 n}(p)}\left[\sum_{j=0}^{n-2}\left[(2 n-1) a_{l}+2(j+1) a_{l+1}\right] x_{p}^{2 j+1}+(2 n-1) a_{n-1} x_{p}^{2 n-1}\right] \\
& =\frac{-1}{\phi^{2 n+1}(p)} \sum_{j=0}^{n-1} \beta_{j} x_{p}^{2 j+1}
\end{aligned}
$$

where $\beta_{l} \geqq 0 ; j=0, \cdots, n-1$. Consequently

$$
\begin{aligned}
\phi^{(2 n+2)}(p) & =\frac{-1}{\phi^{2 n+1}(p)}\left[2 n \sum_{j=0}^{n-1} \beta_{j} x_{p}^{2 j+2}+\sum_{j=0}^{n-1}(2 j+1) \beta_{,} x_{p}^{2 \prime}\right] \\
& =\frac{-1}{\phi^{2 n+1}(p)} \sum_{j=0}^{n+1} \gamma_{j} x_{p}^{2 l}
\end{aligned}
$$

where $\gamma_{\jmath} \geqq 0 ; j=0, \cdots, n+1$. This concludes the proof of the proposition.

Proposition A.2. If for $n=1,2, \cdots$ we define $p_{n}$ by

$$
\exp \left(-\frac{1}{2} x_{p_{n}}^{2}\right)=1 / n \text { and } p_{n} \leqq \frac{1}{2} \text {, }
$$

then there exists $n_{0}$ s.t. for any $n \geqq n_{0}$

$$
p_{n} \leqq p \leqq p_{n}^{\prime} \Rightarrow \phi(p) / \sqrt{n} \leqq \min \left(p, p^{\prime}\right) .
$$

Proof. First, by our definition of $x_{p} ; p=(1 / \sqrt{2 \pi}) \int_{-\infty}^{x_{p}} \exp \left(-\frac{1}{2} x^{2}\right)$ we have

$$
p_{n} \leqq p \leqq p_{n}^{\prime} \Leftrightarrow x_{p}^{2} \leqq x_{p_{n}}^{2} \Leftrightarrow \exp \left(-\frac{1}{2} x_{p}^{2}\right) \geqq \exp \left(-\frac{1}{2} x_{p_{n}}^{2}\right),
$$

hence $p_{n} \leqq p \leqq p_{n}^{\prime} \Leftrightarrow \exp \left(-\frac{1}{2} x_{p}^{2}\right) \geqq 1 / n$ may now be written as

$$
\begin{aligned}
\exp \left(-\frac{1}{2} x_{p}^{2}\right) & \geqq \frac{1}{n} \Rightarrow \frac{1}{\sqrt{n}} \frac{1}{\sqrt{2 \pi}} \exp \left(-\frac{1}{2} x_{p}^{2}\right) \\
& \leqq \min \left(\frac{1}{\sqrt{2 \pi}} \int_{-\infty}^{x_{p}} \exp \left(-\frac{1}{2} x^{2}\right) d x, \frac{1}{\sqrt{2 \pi}} \int_{x_{p}}^{\infty} \exp \left(-\frac{1}{2} x^{2}\right) d x\right) .
\end{aligned}
$$


The statement of the right hand side is

$$
\frac{1}{\sqrt{n}}=\frac{1}{\sqrt{2 \pi}} \exp \left(-\frac{1}{2} x_{p}^{2}\right) \leqq \frac{1}{\sqrt{2 \pi}} \int_{-\infty}^{-\left|x_{p}\right|} \exp \left(-\frac{1}{2} x^{2}\right) d x .
$$

We may therefore consider just, say, $x_{p} \leqq 0$ and prove (replacing $x_{p}$ by $y$ )

$$
\frac{1}{\sqrt{n}} \frac{1}{\sqrt{2 \pi}} \exp \left(-\frac{1}{2} y^{2}\right) \leqq \frac{1}{\sqrt{2 \pi}} \int_{-\infty}^{y} \exp \left(-\frac{1}{2} x^{2}\right) d x
$$

whenever $\exp \left(-\frac{1}{2} y^{2}\right) \leqq 1 / n$ and $y \leqq 0$.

Now (A.6) is true (for all $n \geqq 1$ ) whenever $-1 \leqq y \leqq 0$. This is because it is true for $y=-1$ (direct computation), and the left hand side is concave on $-1 \leqq y \leqq 0$ and has a smaller slope than the right hand side which is convex on $-1 \leqq y \leqq 0$ (see Fig. 1). For $y<-1 ; d / d y\left[\exp \left(-\frac{1}{2} y^{2}\right)\right]$ is positive and increasing hence at any point $y<-1$, the part of the tangent left to $y$ lies below the line $\exp \left(-\frac{1}{2} x^{2}\right)$. It intersects the coordinates axis at $y+1 / y$ (Fig. 2). The integral on the right hand side of (A.6) can then be bounded by

$$
\int_{-\infty}^{y} \exp \left(-\frac{1}{2} x^{2}\right) d x \geqq-\frac{1}{2 y} \exp \left(-\frac{1}{2} y^{2}\right) .
$$

It suffices therefore to prove that

$$
\exp \left(-\frac{1}{2} y^{2}\right) \geqq 1 / n \Rightarrow-1 / 2 y \geqq 1 / \sqrt{n} .
$$

In fact

$$
\exp \left(-\frac{1}{2} y^{2}\right) \geqq 1 / n \Rightarrow|y|=-y \leqq \sqrt{2 \log n} \Rightarrow-1 / 2 y \geqq 1 / 2 \sqrt{2 \log n}
$$

and since $(\log n) / n \rightarrow 0$ let $n_{0}$ be s.t. $n \geqq n_{0} \Rightarrow-1 / 2 y \geqq 1 / \sqrt{n}$ and we have thus proved (A.4) for $n \geqq n_{0}$.

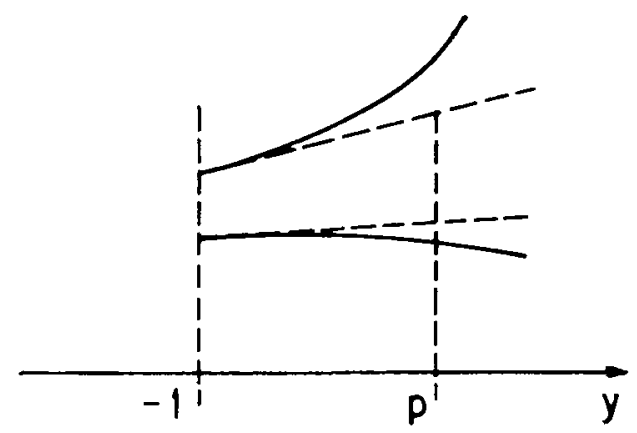

Fig. 1. 


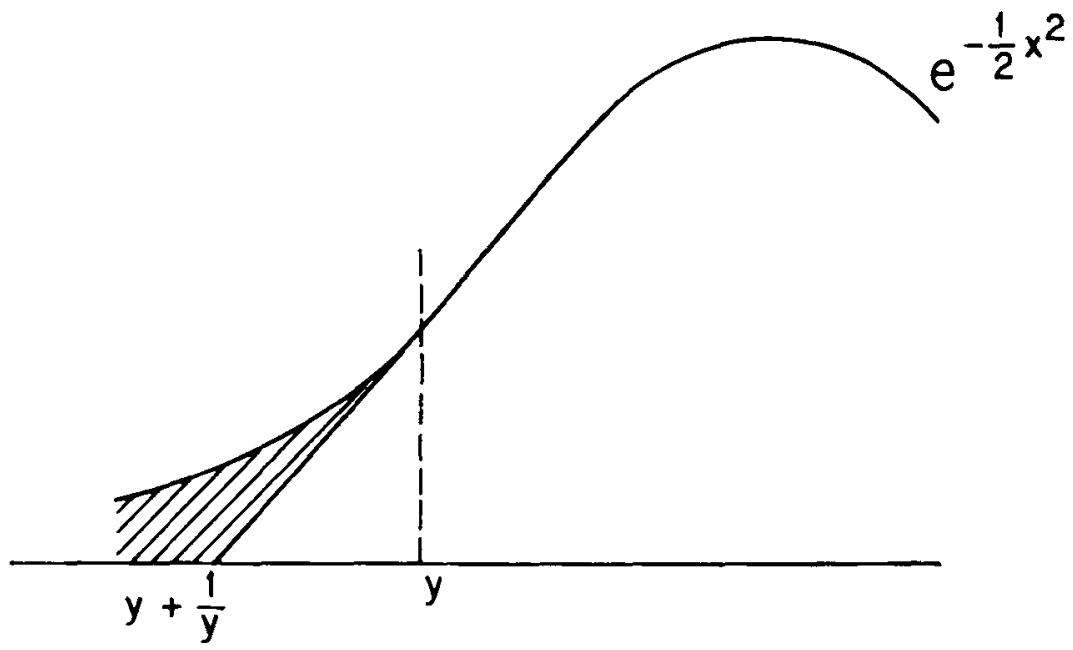

Fig. 2.

Proof of Lemma 4.4. Using Proposition A.1 we expand the first term in the left hand side of $(4.10)$ as follows:

$$
\frac{1}{2}(\phi(p+x)+\phi(p-x))=\phi(p)+\frac{x^{2}}{2} \phi^{\prime \prime}(p)+\frac{x^{4}}{4 !} \cdot \frac{1}{2}\left(\phi^{(4)}(p+\delta)+\phi^{(4)}(p-\delta)\right)
$$

$$
=\phi(p)-\frac{x^{2}}{2 \phi(p)}-\frac{x^{4}}{4 !} \cdot \frac{1}{2}\left(\frac{1+2 x_{p+\delta}^{2}}{\phi^{3}(p+\delta)}+\frac{1+2 x_{p-\delta}^{2}}{\phi^{3}(p-\delta)}\right)
$$

where $0 \leqq \delta \leqq x$.

Clearly it is enough to prove (4.10) for $n \geqq n_{0}$ for fixed $n_{0}$ and then modify the constant $c$ to make (4.10) true for all $n$.

Define $p_{n}$ by $\exp \left(-\frac{1}{2} x_{p_{n}}^{2}\right)=1 / n$ and $p_{n} \leqq \frac{1}{2}$ then by Proposition A.2, $x=$ $\phi(p) / \sqrt{n}$ is in the domain of maximization in (4.10) for $n \geqq n_{0}$, hence denoting the left hand side of (4.10) by A, we get by using (A.7) for $x=\phi(p) / \sqrt{n}$

$$
\begin{array}{r}
A \geqq \frac{1}{\sqrt{n+1}}\left\{\frac{\phi(p)}{\sqrt{n}}+\sqrt{n}\left[\phi(p)-\frac{\phi^{2}(p)}{2 n \phi(p)}\right]-\frac{1}{2}\left(\frac{1+2 x_{p+\delta}^{2}}{\phi^{2}(p+\delta)}+\frac{1+2 x_{p-\delta}^{2}}{\phi^{2}(p-\delta)}\right) \frac{\phi^{4}(p)}{4 ! n^{2}}\right\}, \\
A-\phi(p) \geqq \phi(p)\left[\frac{\sqrt{n+1} / 2 \sqrt{n}-\sqrt{n+1}}{\sqrt{n+1}}\right] \\
-\frac{1}{2}\left(\frac{1+2 x_{p+\delta}^{2}}{\phi^{3}(p+\delta)}+\frac{1+2 x_{p-\delta}^{2}}{\phi^{3}(p-\delta)}\right) \frac{\phi^{4}(p)}{4 ! n^{2} \sqrt{n+1}} .
\end{array}
$$

Since the first term on the right hand side is positive, the second is negative and $\sqrt{n+1} \geqq 1$ we get 


$$
A-\phi(p) \geqq-\frac{1}{2}\left(\frac{1+2 x_{p+\delta}^{2}}{\phi^{3}(p+\delta)}+\frac{1+2 x_{p-\delta}^{2}}{\phi^{3}(p-\delta)}\right) \frac{\phi^{4}(p)}{4 ! n^{2}}
$$

where $0 \leqq \delta \leqq \phi(p) / \sqrt{n}$.

Notice now that $\phi^{(4)}(p)=-\left(1+2 x_{p}^{2}\right) / \phi^{3}(p)$ is a negative and concave function (since $\left.\phi^{(b)}(p) \leqq 0\right)$. Also since $\phi(p)$ is symmetric around $p=\frac{1}{2}\left(\phi(p)=\phi\left(p^{\prime}\right)\right.$ ) and since $x_{p^{\prime}}=-x_{p}, \phi^{(4)}$ is also symmetric around $p=\frac{1}{2}$. It follows that for $p \leqq \frac{1}{2}$

$$
\frac{1}{2}\left(\phi^{(4)}(p-\delta)+\phi^{(4)}(p+\delta)\right) \geqq \phi^{4}(p-\delta),
$$

and by (A.8)

$$
A-\phi(p) \geqq-\frac{1+2 x_{p-\delta}^{2}}{\phi^{3}(p-\delta)} \cdot \frac{\phi^{4}(p)}{4 ! n^{2}}
$$

which is

$$
A-\phi(p) \geqq-\left(1+2 x_{p-\delta}^{2}\right) \frac{1}{\sqrt{2 \pi}} \exp \left(\frac{3}{2} x_{p-\delta}^{2}-2 x_{p}^{2}\right) \frac{1}{4 ! n^{2}} .
$$

Now $1+2 x^{2} \leqq 8 \exp \left(x^{2} / 4\right)$ for $-\infty<x<\infty$ hence

$$
A-\phi(p) \geqq \frac{8}{\sqrt{2 \pi} 4 ! n^{2}} \exp \left(\frac{7}{4} x_{p-\delta}^{2}-2 x_{p}^{2}\right) .
$$

We now establish the existence of a constant $\tilde{K}$ such that $x_{p}-x_{p-\delta} \leqq \tilde{K} / \sqrt{n}$ holds for $p_{n} \leqq p \leqq p_{n}^{\prime}, p \leqq \frac{1}{2}$ and $n$ sufficiently large. Since $0 \leqq \delta \leqq \phi(p) / \sqrt{n}$ and since $x_{p}-x_{p-\delta}$ is monotonically increasing with $\delta$ we have to show that $\Delta \leqq \tilde{K} / \sqrt{n}$ where $\Delta=x_{p}-x_{p-\phi(p) / n}$. Letting $y=x_{p} \leqq 0$ we claim in other words that $\phi(p) / \sqrt{n}=\int_{y-\Delta}^{y}(1 / \sqrt{2 \pi}) \exp \left(-\frac{1}{2} x^{2}\right) d x$ implies $\Delta \leqq \tilde{K} / \sqrt{n}$.

In fact for $-1 \leqq y \leqq 0$ we have

$$
1 / \sqrt{2 \pi n} \geqq \phi(p) / \sqrt{n}=\int_{y-\Delta}^{y} \frac{1}{\sqrt{2 \pi}} \exp \left(-\frac{1}{2} x^{2}\right) d x \geqq \frac{1}{\sqrt{2 \pi e}} \Delta,
$$

which implies $\Delta \leqq \sqrt{e} / \sqrt{n}$.

For $y \leqq-1$ the tangent to $(1 / \sqrt{2 \pi}) \exp \left(-\frac{1}{2} x^{2}\right)$ at $x=y$ lies below the function and intersects the $x$ axis at $y+1 / y$ (see Fig. 3) forming a triangular area $(-1 / 2 y)(1 / \sqrt{2 \pi}) \exp \left(-\frac{1}{2} y^{2}\right)=\phi(p) / 2|y|$.

Now $p \geqq p_{m}$ implies $|y|=\left|x_{p}\right| \leqq\left|x_{p_{n}}\right|=\sqrt{2 \log n} \leqq \frac{1}{2} \sqrt{n}$ for $n$ sufficiently large, hence the triangular area is $\geqq \phi(p) / \sqrt{n}$ which implies $\Delta \leqq 1 /|y|$. The area of the shaded trapezoid is $\phi(p)(2-|y| \Delta) \Delta / 2$, therefore

$$
\frac{\phi(p)}{\sqrt{n}}=\frac{1}{\sqrt{2 \pi}} \int_{y-\Delta}^{v} \exp \left(-\frac{1}{2} x^{2}\right) d x \geqq \phi(p)(2-|y| \Delta) \frac{\Delta}{2} \geqq \phi(p) \cdot \frac{\Delta}{2} .
$$




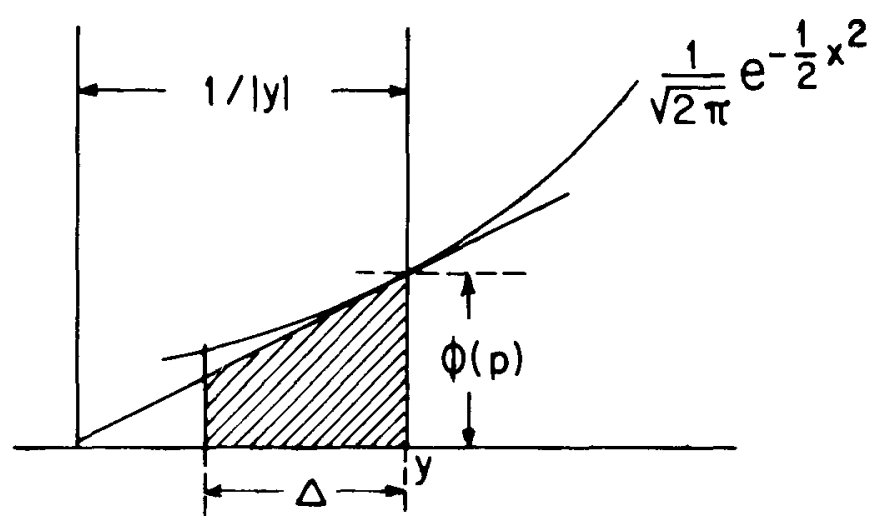

Fig. 3.

This completes the proof of $\Delta \leqq \tilde{K} / \sqrt{n}$ for a suitable constant $\tilde{K}$ and $n$ sufficiently large. From here we get

and

$$
x_{p-\delta}^{2}=\left(x_{p}-\Delta\right)^{2} \leqq\left(x_{p}-\frac{\tilde{K}}{\sqrt{n}}\right)^{2}=x_{p}^{2}-\frac{2 \tilde{K} x_{p}}{\sqrt{n}}+\frac{\tilde{K}^{2}}{n},
$$

$$
\frac{7}{4} x_{p-8}^{2}-2 x_{p}^{2}=-\frac{1}{4} x_{p}^{2}-7 \tilde{K} x_{p} / 2 \sqrt{n}+7 \tilde{K}^{2} / 4 n .
$$

Since $x_{p}<0$, the right hand side has a maximum (with respect to $n$ ) at $n_{0}$, hence

$$
{ }_{4}^{7} x_{p-8}^{2}-2 x_{p}^{2} \leqq-\frac{1}{4} x_{p}^{2}-7 \tilde{K} x_{p} / 2 \sqrt{n_{0}}+7 \tilde{K}^{2} / 4 n_{0} \leqq K
$$

where $K$ is the maximum of the parabola (in $x_{p}$ ) at the right hand side. Combining (A.13) and (A.10) we finally obtain the existence of a constant $C_{1}>0$ s.t.

$$
A \geqq \phi(p)-C_{1} / 2 \text { for } n \geqq n_{0} \text { and } p_{n} \leqq p \leqq p_{n}^{\prime} .
$$

It remains to establish (A.14) also for $p \leqq p_{n}$ or $p \geqq p_{n}^{\prime}$. In this case, by the definition of $p_{n}: \exp \left(-\frac{1}{2} x_{p}^{2}\right) \leqq 1 / n$ and therefore $\phi(p) \leqq 1 / n \sqrt{2 \pi}$. So (choosing $x=0$ )

$$
\begin{aligned}
& \frac{1}{\sqrt{n+1}} \operatorname{Max}_{\substack{0 \leqq x \leqq p \\
0 \leqq x \leqq p^{\prime}}}\left[\sqrt{n} \frac{1}{2}(\phi(p+x)+\phi(p-x))+x\right] \geqq \frac{\sqrt{n}}{\sqrt{n+1}} \phi(p) \\
& \geqq \phi(p)-\phi(p)(1-\sqrt{n} / \sqrt{n+1}) \geqq \phi(p)-\phi(p) /(2 n+1) \\
& \geqq \phi(p)-1 / n(2 n+1) \sqrt{2 \pi} \geqq \phi(p)-C_{2} / n^{2}
\end{aligned}
$$

for some constant $C_{2}$. 
Choose now $C_{3}$ s.t. $A \geqq \phi(p)-C_{3} / n^{2}$ for $1 \leqq n \leqq n_{0}$ and finally choose $c=\max \left(C_{1}, C_{2}, C_{3}\right)$. This completes the proof of Lemma 4.4 .

Proof of Lemma 4.5. We have to prove the existence of a constant $K>0$ s.t. for $0 \leqq p \leqq 1$

$$
\text { (A.15) } \begin{aligned}
& \frac{1}{\sqrt{n+1}} \operatorname{Max}_{(\xi, \eta) \in S(p)}\left[\sqrt{n} \frac{\eta}{\xi+\eta} \phi(p+\xi)+\sqrt{n} \frac{\xi}{\xi+\eta} \phi(p-\eta)+\frac{2 \xi \eta}{\xi+\eta}\right] \\
& \leqq \phi(p)+K / n^{2}
\end{aligned}
$$

where $S(p)=\left\{(\xi, \eta) \mid 0 \leqq \xi \leqq p^{\prime} ; 0 \leqq \eta \leqq p\right\}$.

Since $\phi$ is continuous and $S(p)$ is compact, the maximum in (A.15) is achieved, say, at $\left(\xi_{0}, \eta_{0}\right)$. From $(d \phi / d p)_{1^{-}}=-\infty ;(d \phi / d p)_{0_{+}}=+\infty$ it follows that $\xi_{0} \neq p^{\prime}$ and $\eta_{0} \neq p$. Furthermore we claim that if $p p^{\prime} \neq 0$ then $\xi_{0} \neq 0$ and $\eta_{0} \neq 0$. In fact, denote the function to be maximized in (A.15) by $F(\xi, \eta)$, then $F(0, \eta)=F(\xi, 0)=\sqrt{n} \phi(p)$ while

$$
\left.\operatorname{Max}_{(\xi, n) \in S(p)} F(\xi, \eta) \geqq \operatorname{Max}_{0 \leq x \leq p \wedge p^{\prime}} F(x, x)=\sqrt{n} \phi(p)+\operatorname{Max}_{0 \leq x \leq p \wedge p^{\prime}}\left[O\left(x^{2}\right)+x\right]\right)>\sqrt{n} \phi(p) .
$$

We conclude that $\left(\xi_{0}, \eta_{0}\right)$ is a local maximum of $F(\xi, \eta)$ in $S(p)$. Equating first partial derivatives to 0 yields

$$
-\frac{\eta_{0}}{\left(\xi_{0}+\eta_{0}\right)^{2}} \sqrt{n} \phi\left(p+\xi_{0}\right)+\frac{\eta_{0}}{\xi_{0}+\eta_{0}} \sqrt{n} \phi^{\prime}\left(p+\xi_{0}\right)
$$

$$
\begin{gathered}
+\frac{\eta_{0}}{\left(\xi_{0}+y_{0}\right)^{2}} \sqrt{n} \phi\left(p-\eta_{0}\right)+\frac{2 \eta_{0}^{2}}{\left(\xi_{0}+\eta_{0}\right)^{2}}=0, \\
\frac{\xi_{0}}{\left(\xi_{0}+\eta_{0}\right)^{2}} \sqrt{n} \phi(p+\xi)-\frac{\xi_{0}}{\left(\xi_{0}+\eta_{0}\right)^{2}} \sqrt{n} \phi(p-\eta)
\end{gathered}
$$

(A.17)

$$
-\frac{\xi_{0}}{\xi_{0}+\eta_{0}} \sqrt{n} \phi^{\prime}\left(p-\eta_{0}\right)+\frac{2 \xi_{0}^{2}}{\left(\xi_{0}+\eta_{0}\right)^{2}}=0
$$

Dividing (A.16) by $\eta_{0} /\left(\xi_{0}+\eta_{0}\right)$, (A.17) by $\xi_{0} /\left(\xi_{0}+\eta_{0}\right)$ and adding the results we get $\sqrt{n}\left[\phi^{\prime}\left(p+\xi_{0}\right)-\phi^{\prime}\left(p-\eta_{0}\right)\right]+2=0$.

Recalling that $\phi^{\prime}(p)=-x_{p}$ we have

$$
x_{p+\xi_{0}}-x_{p-n_{0}}=2 / \sqrt{n} \text {. }
$$

By the mean value theorem

$$
x_{p+\xi_{0}}-x_{p-\eta_{0}}=\left[\left(p+\xi_{0}\right)-\left(p-\eta_{0}\right)\right] x_{\left(\theta\left(p+\xi_{0}\right)+(1-\theta)\left(p-\eta_{0}\right)\right)}^{\prime}
$$

for some $0 \leqq \theta \leqq 1$. 
Using (A.18) and recalling that $x^{\prime}(p)=1 / \phi(p)$ we get

$$
\xi_{0}+\eta_{0}=(2 / \sqrt{n}) \phi\left[\theta\left(p+\xi_{0}\right)+(1-\theta)\left(p-\eta_{0}\right)\right]
$$

Now

$$
\begin{aligned}
\frac{\phi\left[\theta\left(p+\xi_{0}\right)+(1-\theta)\left(p-\eta_{0}\right)\right]}{\phi(p)} & =\exp \left\{-\frac{1}{2}\left[x_{p}^{2}-x_{p+\theta \xi_{0}-(1-\theta) \eta_{0}}^{2}\right]\right\} \\
& =\exp \left\{-\frac{1}{2}\left[x_{p}+x_{p+\theta \xi_{0}-(1-\theta) \eta_{0}}\right]\left[x_{p}-x_{p+\theta 5_{0}-(1-\theta) \eta_{0}}\right]\right\}
\end{aligned}
$$

Since $x_{p}$ is monotonically increasing in $p$ we get from (A.20)

$$
\left[x_{p}+x_{\left.p+\theta \xi_{1}-(1-\theta) m_{1}\right)}\right]\left[x_{p}-x_{p+\theta \xi_{0}-(1-\theta) m_{0}}\right] \leqq(2 / \sqrt{n})\left(2\left|x_{p}\right|+2 / \sqrt{n}\right)
$$

and by (A.19) and (A.20) therefore

$$
\xi_{0}+\eta_{0} \leqq(2 / \sqrt{n}) \phi(p) \exp \left(2\left|x_{p}\right| / \sqrt{n}\right) \cdot \exp (2 / n) .
$$

Denote

$$
G(\xi, \eta)=\frac{\eta}{\xi+\eta} \phi(p+\xi)+\frac{\xi}{\xi+\eta} \phi(p-\eta) .
$$

Expanding $\phi(p+\xi)$ and $\phi(p-\eta)$ yields the following expansion for $G(\xi, \eta)$ :

$$
G(\xi, \eta)=\phi(p)+\frac{1}{2} \xi \eta \phi^{\prime \prime}(p)+\frac{1}{6} \xi \eta(\xi-\eta) \phi^{\prime \prime \prime}(p)+\frac{1}{24} \xi \eta\left(\xi^{2}-\xi \eta+\eta^{2}\right) \phi^{(4)}(p)
$$

$$
+\frac{1}{120}\left[\frac{\eta \xi^{5}}{\xi+\eta} \phi^{(5)}\left(p+\sigma_{1} \xi\right)-\frac{\xi \eta^{5}}{\xi \eta} \phi^{(5)}\left(p-\sigma_{2} \eta\right)\right]
$$

where $0 \leqq \sigma_{1} \leqq 1$ and $0 \leqq \sigma_{2} \leqq 1$.

First consider the last term in (A.22) which we denote by $K(p ; \xi, \eta)$. Since $\phi^{(3)}$ is decreasing we have by Proposition A.1

$$
K(p ; \xi, \eta) \leqq-\frac{1}{120} \xi \eta\left(\xi^{2}+\eta^{2}\right)(\xi-\eta) x_{p}\left(7+6 x_{p}^{2}\right) / \phi^{4}(p) .
$$

By (A.21), since $\max \left(\xi \eta, \xi^{2}+\eta^{2}\right) \leqq(\xi+\eta)^{2}$ and $\xi-\eta \leqq \xi+\eta$ we have

$$
\left|K\left(p ; \xi_{0}, \eta_{0}\right)\right| \leqq \frac{1}{120}\left[\frac{2}{\sqrt{n}} \phi(p) \exp \left(2\left|x_{p}\right| / \sqrt{n}\right)+\frac{2}{\sqrt{n}}\right]^{5} x_{p}\left(7+6 x_{p}^{2}\right) / \phi^{4}(p)
$$

$(\mathrm{A} .24)=\frac{4}{15 \sqrt{n^{5}}}\left[\phi(p) x_{p}\left(7+6 x_{p}^{2}\right) \exp \left(10\left|x_{p}\right| / \sqrt{n}\right)+10 / \sqrt{n}\right]$

$$
=\frac{4}{15 \sqrt{2 \pi n^{5}}}\left[x_{p}\left(7+6 x_{p}^{2}\right) \exp \left(10\left|x_{p}\right| / \sqrt{n}+10 / \sqrt{n}-\frac{1}{2} x_{p}^{2}\right)\right] .
$$


The last expression is clearly a bounded function of $x_{p}$, hence

$$
\left|K\left(p ; \xi_{0}, \eta_{0}\right)\right| \leqq K_{1} / n^{2}
$$

for some constant $K_{1}$.

By (A.22) and (A.25), using Proposition A.1 we obtain

$$
\begin{gathered}
G\left(\xi_{0}, \eta_{0}\right) \leqq \phi(p)-\xi_{0} \eta_{0} / 2 \phi(p)-\frac{1}{6} \xi_{0} \eta_{0}\left(\xi_{0}-\eta_{0}\right) x_{p} / \phi^{2}(p)- \\
-\frac{1}{24} \xi_{0} \eta_{0}\left(\xi_{0}^{2}-\xi_{0} \eta_{0}+\eta_{0}^{2}\right)\left(1+2 x_{p}^{2}\right) / \phi^{3}(p)+K_{1} / n^{2} .
\end{gathered}
$$

Therefore

$$
\text { (A.27) } \operatorname{Max}_{(\xi, \eta) \in S(p)}\left[G(\xi, \eta)+\frac{1}{\sqrt{n}} \frac{2 \xi \eta}{\xi+\eta}\right] \leqq \phi(p)+K_{1} / n^{2}+\operatorname{Max}_{(\xi, \eta) \in S(p)} D(\xi, \eta)
$$

where

$$
D(\xi, \eta)=\frac{1}{\sqrt{n}} \frac{2 \xi \eta}{\xi+\eta}-\frac{\xi \eta}{2 \phi(p)}-\frac{1}{6} \xi \eta(\xi-\eta) \frac{x_{p}}{\phi^{2}(p)}-\frac{1}{24} \xi \eta\left(\xi^{2}-\xi \eta+\eta^{2}\right) \frac{1+2 x_{p}^{2}}{\phi^{3}(p)}
$$

Observe that $D(0, \eta)=D(\xi, 0)=0 ; D(\varepsilon, \varepsilon)>0$ for $\varepsilon>0$ sufficiently small. Also $D(\xi, \eta) \rightarrow-\infty$ as $\xi \rightarrow \infty$ or $\eta \rightarrow \infty$. It follows that $D$ restricted to the nonnegative orthant has a global maximum which is also a local maximum. Equating first derivatives of $D(\xi, \eta)$ to 0 gives

$$
\begin{aligned}
& \text { (A.29) } \begin{aligned}
0=\frac{\partial D}{\partial \xi}=\frac{1}{\sqrt{n}} \frac{2 \eta^{2}}{(\xi+\eta)^{2}}-\frac{\eta}{2 \phi(p)}-\frac{1}{6}[\eta(\xi-\eta) & +\xi \eta] \frac{x_{p}}{\phi^{2}(p)} \\
& -\frac{\eta}{24}\left(3 \xi^{2}-2 \xi \eta+\eta^{2}\right) \frac{1+2 x_{p}^{2}}{\phi^{3}(p)},
\end{aligned} \\
& \text { (A.30) } \begin{aligned}
0=\frac{\partial D}{\partial \eta}=\frac{1}{\sqrt{n}(\xi+\eta)^{2}}-\frac{2 \xi^{2}}{2 \phi(p)}+\frac{1}{6}[\xi(\eta-\xi) & +\xi \eta] \frac{x_{p}}{\phi^{2}(p)} \\
& -\frac{\xi}{24}\left(3 \eta^{2}-2 \xi \eta+\xi^{2}\right) \frac{1+2 x_{p}^{2}}{\phi^{3}(p)} .
\end{aligned}
\end{aligned}
$$

Adding

$$
\frac{1}{\eta} \frac{\partial D}{\partial \xi}+\frac{1}{\xi} \frac{\partial D}{\partial \eta}
$$

we get

$$
\begin{aligned}
& \text { (A.31) } 2 / \sqrt{n}(\xi+\eta)-1 / \phi(p)-\frac{1}{2}(\xi-\eta) x_{p} / \phi^{2}(p)-\frac{1}{6}\left(\xi^{2}-\xi \eta+\eta^{2}\right) \\
& \times\left(1+2 x_{p}^{2}\right) / \phi^{3}(p)=0 .
\end{aligned}
$$


Subtracting

we get

$$
\frac{1}{\eta} \frac{\partial D}{\partial \xi}-\frac{1}{\xi} \frac{\partial D}{\partial \eta}
$$

(A.32) $2(\eta-\xi) / \sqrt{n}(\xi+\eta)^{2}-\frac{1}{6}(\xi+\eta) x_{p} / \phi^{2}(p)-\frac{1}{12}\left(\xi^{2}-\eta^{2}\right)\left(1+2 x_{p}^{2}\right) / \phi^{3}(p)=0$.

By dividing (A.32) by $\left(\xi^{2}-\eta^{2}\right)$ and eliminating $(\eta-\xi)$ we obtain

$$
\eta-\xi=\frac{x_{p} / \phi^{2}(p)}{\frac{12}{\sqrt{n}(\xi+\eta)^{3}}+\left(\frac{1}{2}+x_{p}^{2}\right) / \phi^{3}(p)} .
$$

Replacing in (A.31) $\left(\xi^{2}-\xi \eta+\eta^{2}\right)$ by $\frac{1}{4}(\xi+\eta)^{2}+\frac{3}{4}(\xi-\eta)^{2}$, and $(\xi-\eta)$ by its value according to (A.33), we get

$$
\frac{2}{\sqrt{n}(\xi+\eta)}-\frac{1}{\phi(p)}+\frac{x_{p}^{2} / \phi^{4}(p)}{24 / \sqrt{n}(\xi+\eta)^{3}+\left(1+2 x_{p}^{2}\right) / \phi^{3}(p)}-\frac{1}{24} \frac{1+2 x_{p}^{2}}{\phi^{3}(p)}(\xi+\eta)^{2}
$$

$$
-\frac{1}{8} \frac{1+2 x_{p}^{2}}{\phi^{3}(p)}\left[\frac{x_{p} / \phi^{2}(p)}{12 / \sqrt{n}(\xi+\eta)^{3}+\left(\frac{1}{2}+x_{p}^{2}\right) / \phi^{3}(p)}\right]^{2}=0 .
$$

It is easily verified that the expression in (A.34) tends to $+\infty$ as $(\xi+\eta) \rightarrow 0$. On the other hand, the last two terms are always negative and the third is bounded from above by $[1 / \phi(p)]\left\{\operatorname{Max}_{x}\left[x /\left(1+2 x^{2}\right)\right]\right\}$ which is $1 / 2 \sqrt{2} \phi(p)$. So if we denote the left hand side of (A.34) by $L(\xi, \eta)$ we can assert that

$$
L(\xi, \eta) \leqq 2 / \sqrt{(\xi+\eta)}-1 / \phi(p)+1 / 2 \sqrt{2} \phi(p) .
$$

The right hand side of (A.35) is non-negative if and only if

$$
\xi+\eta \leqq \alpha \phi(p) / \sqrt{n} \text { where } \alpha=2 /(1-1 / 2 \sqrt{2}) \sim 3.1 .
$$

It follows therefore from (A.35) and (A.34) that any solution $(\xi, \eta)$ of (A.31) and (A.33) must satisfy

$$
\xi+\eta \leqq \alpha \phi(p) / \sqrt{n} .
$$

By (A.33) we get that at the maximum $(\xi, \eta)$

$$
|\eta-\xi| \leqq \frac{\left|x_{p}\right| / \phi^{2}(p)}{12 n / \phi^{3}(p) \alpha^{3}+\left(\frac{1}{2}+x_{p}^{2}\right) / \phi^{3}(p)}
$$

$$
=\frac{\left|x_{p}\right| \phi(p)}{12 n / \alpha^{3}+\left(\frac{1}{2}+x_{p}^{2}\right)}<\frac{\left|x_{p}\right| \phi(p) \alpha^{3}}{12 n} .
$$


Being interested in obtaining an upper bound for the global maximum of $D$ we replace its last two terms by an upper bound at the maximum. The resulting function will have a maximum which is greater than or equal to that of $D$. Now the last term of $D$ (in (A.28)) is not positive and as for the third term, by (A.36) and (A.37),

$$
\begin{aligned}
\frac{1}{6} \xi \eta(\xi-\eta) \frac{x_{p}}{\phi^{2}(p)} & \leqq \frac{1}{6} \alpha^{2} \frac{\phi^{2}(p)\left|x_{p}\right| \phi(p) \alpha^{3}}{n} \frac{12 n \phi^{2}(p)}{12} \\
& =\frac{\alpha^{5}}{72} \frac{1}{n^{2}} \operatorname{Max}_{x} \frac{1}{\sqrt{2 \pi}}|x| \exp \left(-\frac{1}{2} x^{2}\right) \leqq K_{2} / n^{2} .
\end{aligned}
$$

We conclude that

$$
\operatorname{Max}_{\xi, \eta} D(\xi, \eta) \leqq K_{2} / n^{2}+\operatorname{Max}_{\xi, \eta} D_{1}(\xi, \eta)
$$

where $K_{2}$ is a constant and

$$
D_{1}(\xi, \eta)=\frac{2 \xi \eta}{\xi+\eta} \cdot \frac{1}{\sqrt{n}}-\frac{\xi \eta}{2 \phi(p)}
$$

Equating $\partial D_{1} / \partial \xi$ and $\partial D_{1} / \partial \eta$ to 0 we get

$$
\begin{aligned}
& \frac{1}{\eta} \frac{\partial D_{1}}{\partial \xi}=\frac{2 \eta}{(\xi+\eta)^{2}} \cdot \frac{1}{\sqrt{n}}-\frac{1}{2 \phi}=0, \\
& \frac{1}{\xi} \frac{\partial D_{1}}{\partial \eta}=\frac{2 \xi}{(\xi+\eta)^{2}} \cdot \frac{1}{\sqrt{n}}-\frac{1}{2 \phi}=0
\end{aligned}
$$

which imply $\xi=\eta=\phi / \sqrt{n}$ and hence

$$
\operatorname{Max}_{(\xi, \eta) \in S(p)} D_{1}(\xi, \eta) \leqq \phi(p) / n-\phi(p) / 2 n=\phi(p) / 2 n .
$$

By (A.27), (A.38) and (A.40)

$$
\operatorname{Max}_{(\xi, \eta) \in S(p)}\left[G(\xi, \eta)+\frac{1}{\sqrt{n}} \frac{2 \xi \eta}{\xi+\eta}\right] \leqq \phi(p)(1+1 / 2 n)+\left(K_{1}+K_{2}\right) / n^{2} .
$$

Coming back to (A.15) we have now by (A.41)

$$
\frac{1}{\sqrt{n+1}} \operatorname{Max}_{(\xi, \eta) \in S(p)}\left[\sqrt{n} \frac{\eta}{\xi+\eta} \phi(p+\xi)+\sqrt{n} \frac{\xi}{\xi+\eta} \phi(p-\eta)+\frac{2 \xi \eta}{\xi+\eta}\right]
$$

$$
=\frac{\sqrt{n}}{\sqrt{n+1}} \operatorname{Max}_{(\xi, \eta) \in S(p)}\left[G(\xi, \eta)+\frac{1}{\sqrt{n}} \cdot \frac{2 \xi \eta}{\xi+\eta}\right] \leqq \phi(p)\left(1+\frac{1}{2 n}\right) \frac{\sqrt{n}}{\sqrt{n+1}}+\frac{K_{1}+K_{2}}{n^{2}} .
$$


Now notice that $\sqrt{1+1 / n} \geqq 1+1 / 2 n-1 / 8 n^{2}$, therefore

$$
\left(1+\frac{1}{2 n}\right) \frac{\sqrt{n}}{\sqrt{n+1}}-1=\frac{1+1 / 2 n}{\sqrt{1+1 / n}}-1 \leqq \frac{1 / 8 n^{2}}{1+1 / 2 n-1 / 8 n^{2}} \leqq K_{3} / n^{2}
$$

where $K_{3}$ is a constant. It follows that

$$
\text { (A.43) } \begin{aligned}
\phi(p)\left(1+\frac{1}{2 n}\right) \sqrt{n} / \sqrt{n+1}+K_{1} / n^{2} \leqq \phi(p)+\phi(p) K_{2} / n^{2}+ & K_{1} / n^{2} \\
& \leqq \phi(p)+K / n^{2}
\end{aligned}
$$

where $K$ is a constant. (A.42) and (A.43) conclude the proof of Lemma 4.5.

\section{REFERENCES}

1. J. L. Doob, Stochastic Processes, John Wiley and Sons Inc., New York, 1953.

2. W. Feller, An Introduction to Probability Theory and ts Applications I (third edition), John Wiley and Sons Inc., New York, 1968.

3. J. F. Mertens and S. Zamir, The normal distribution and repeated games, to appear in Internat. J. Game Theory (1977).

Université Catholique de Louvain

LOUVAIN, BELgIUM

AND

The Hebrew Universtty of Jerusalem

JERUSALEM, ISRAEL 